\title{
Reciprocating compressor optimum configuration with respect to performance, reliability and commercial conditions
}

\author{
A. Almasi \\ Rotating Equipment Department, Tecnicas Reunidas S.A., Spain
}

\begin{abstract}
Reciprocating compressors are flexible in order to handle wide capacity and condition swings, offer a very efficient method of compressing almost any gas mixture over a wide range of pressure, can generate a high head independent of density, and have numerous applications and wide power ratings. These make them vital components in various units of industrial plants. In this paper the optimum reciprocating compressor configuration regarding inter-stage pressures, the low suction pressure, non-lubricated cylinder, speed of the machine, capacity control system, compressor valve, lubrication system, piston rod coating, liner material, barring device, pressure drops, rod load, pin reversal, discharge temperature, cylinder coolant system, performance, flow, coupling, flywheel, special tools, condition monitoring (including vibration, thermal and rod drop monitoring), commercial points, delivery and acoustic conditions are presented. Keywords: reciprocating compressor, optimum configuration, evaluation, performance, reliability.
\end{abstract}

\section{Introduction}

Reciprocating compressors are the most common type of compressors found in industrial applications [1-4]. Worldwide installed reciprocating compressor horsepower is approximately three times that of centrifugal compressors and the maintenance costs of reciprocating compressors are approximately three and half times greater than those for centrifugal compressors [3]. The evaluation and purchase of a reciprocating compressor involves an optimization process with respect to the full array of compressor data. Otherwise, equipment may be purchased that is not be suitable over the full range of operating cases, reliability levels, commercial terms or power requirement for the client's needs. 


\section{Reciprocating compressor optimum configuration}

\subsection{Machine design and selection}

\subsubsection{Inter-stage design pressures}

The discharge pressure of each stage is normally protected by pressure relief valves; high pressure discharge switches are seldom seen [2]. Optimum interstage pressures can be obtained by the formulation and optimization of performance and investment for the compressor and inter-stage facilities. Some vendors intend to change the inter-stage pressures, with generally higher pressures during the early stages and a lower pressure ratio during the final stages. Vendor-offered inter-stage pressures based on only the compressor without respect to inter-stage facilities are not justified. Inter-stage pressures are going to increase during part load operations and high suction pressure. If this is not acceptable, the additional clearance pocket on the first stage cylinder and the part load operation inhabitation by the controlling logic can be studied. Generally it is a compromise to fix inter-stage facility design pressure based on vendor-recommended PRV set points (around 10-15\% higher due to part load operation and suction pressure variations).

\subsubsection{Low suction pressure with full flow}

Sometimes, due to process requirements, reciprocating compressors are capable of operating at the lowest suction pressure and full design flow at normal discharge pressure. This can have a strong effect on compressor sizing, especially the frame rating and motor power (a power increase of more than $35 \%$ for $20 \%$ suction below normal). It is optimum to respect this condition as a design point.

\subsubsection{Minimum speed-lubricated cylinder}

A reliable machine involves low speed (around 350 RPM) and a lubricated cylinder. The optimum piston speed is $3-4.4 \mathrm{~m} / \mathrm{s}$. The optimum configuration is horizontal cylinder(s) with a discharge nozzle on the bottom side. For small compressors some vendors intend to deviate the lubricated cylinder or low speed. The optimum option is a lubricated cylinder with the available lowest speed machines. Probably less than $20 \%$ of all reciprocating compressors are designed for non-lubricated operation, just because of process demands (oxygen, high pressure air, etc) [2].

\subsubsection{Unloader and capacity control system}

The stepless capacity control system uses a finger type unloader, is pneumatically actuated, and unloads the suction valve for only a portion of the compression cycle to achieve the adjusted capacity [2]. Finger type unloaders have the potential for damaging the valve sealing elements and require more care for maintenance [2]. Valves and unloaders cause around $44 \%$ of unscheduled reciprocating shut downs $[5,6]$ and this selection has a strong effect on reliability [5-8]. For small machines, $100 \%$ spill back is the optimum solution, because power is low. For big machines, optimum configuration is the selection of part load steps based on a plug/port unloader and clearance pocket. 


\subsubsection{Condition monitoring}

Condition monitoring [9-13] is particularly cost effective and includes necessary items to identify malfunctions at an early stage (lower maintenance costs and a lower risk of accidents). Optimum vibration monitoring is as follows: 1Vibration - continuous monitoring (shut down). Velocity transducers are preferred over accelerometers due to a better signal to noise ratio [9]. The optimum configuration is each end of the crankcase about halfway up from the base plate in line with a main bearing [9]. 2- Each cross head accelerometer (alarm). 3- Electric motor vibration (shut down). Optimum temperature monitoring is as follows: 1- high gas discharge temperature - each cylinder (alarm and shutdown); 2- pressure packing case - piston rod temperature (alarm); 3- High cross head pin temperature (alarm); 4- high main and motor bearing temperature (alarm); 5- valve temperature (monitoring); 6- oil temperature out of frame (alarm); 7- high jacket water temperature - each cylinder (alarm). Optimum implementation is properly set trip levels that are just high enough over the normal operating levels to detect mechanical failures, but not so high as to miss the failure prior to catastrophic release [9]. Proximity probes are typically located under the piston rods [9] and used to measure the rod position and determine the wear of the piston and rider bands, malfunction, e.g. a cracked piston rod attachment, a broken crosshead shoe, or even a liquid carryover to a cylinder. The optimum figure is just for the alarm and not for a shut down. Optimum cold run outs and normal condition operating run outs are about 50 microns ( 2 mils) and on the order of 50 to 150 microns ( 2 to 6 mils) peak to peak, respectively [9]. All shutdown functions shall be two out of three voting to avoid unnecessary trips. Usually this can be deviated for compressor frame vibration and temperature-related trips.

\subsubsection{Valve selection}

Cylinder valves are the most critical components of reciprocating compressors and strongly influence the reliability and efficiency $[5-8,10]$. Valve defects are obviously responsible for most of the unscheduled maintenance events $[5-8,10]$. Three main valve types are ring type, ported plate and poppet. For big machines (generally low speed and high pressure ratios) and small machines (relatively higher speeds) ring type valves and plate type valves are the optimum choice, respectively. The optimum valve size shall be obtained with respect to efficiency, reliability and performance requirements including minimum clearance volume. Lift is the distance travelled by the valves moving elements. The higher the lift, the higher the valve flow area, the lower the valve pressure drop, the less consumed power, the higher the moving elements impact velocities and the lower the valve durability. An acceptable compromise should be found. Optimum valve spring stiffness is also important. Too much spring stiffness can lead to valve flutter (more compressor power and considerable wear rate) or early closing of the valve (reduced capacity). Too little spring stiffness causes late valve closing and reverse flow (higher velocity, less reliability and reduced capacity). Nonlinear partial differential equations describing the valve differential pressure and the valve element motion (such as [14]) can be used in 
the optimization process to estimate the optimum valve lift, spring stiffness and gas velocity for each machine and application.

\subsubsection{Piston rod coating}

The Piston rod seal is the second most important area for the reliability of the reciprocating compressor and the most likely path for potentially hazardous process gas leakage [8]. The packing life could be improved three times by adding the proper tungsten carbide piston rod coating [10], which is the optimum selection.

\subsubsection{Cylinder liner material}

The cylinder liner is used to provide a renewable wearing surface. The liners made by Ni-Resist cast iron (high Nickel content) are not recommended due to problems such as permanent distortions. The optimum selection is grey cast iron [8], except in very high pressure or extremely high corrosive applications.

\subsubsection{Passive vibration reduction system}

Sometimes an odd number of cylinders is avoidable. In this case a dummy crosshead shall be used to reduce vibration. The spring-mass-spring system shall also be studied for passive force counter balance and a further reduction in vibration, where the dummy crosshead is, on the one hand, flexibly attached to a movable piston assembly and on the other hand, to the stationary compressor casing using auxiliary mechanical springs.

\subsubsection{Future expansion}

Future expansion planning can save money and time if the process changes (capacity increase, molecular weight increase due to catalyst change, etc) are foreseen [12]. The optimum selection is sizing cylinders for economical operation at the present rate; the frame can be sized for future applications. When the future conditions become a reality, the cylinders can be changed while keeping the same frame. Generally it is optimum to over size the journal diameter to include margins for future development, thus ensuring that crankshaft size would never become the first important limitation of the design [15].

\subsection{Commercial optimum conditions}

\subsubsection{Commercial conditions}

It is absolutely necessary to receive at least three proposals and have, as a minimum, two technically accepted machines. It is completely justifiable to extend the proposal deadline, extend the clarification time, accept an optimum configuration, allow reasonable deviations and attend extensive clarification meetings to have at least two clarified and technically accepted proposals.

\subsubsection{Delivery}

Small and medium machines are delivered fully fabricated as one skid mounted package. For very big machines, the optimum situation is to deliver the machine prefabricated (including the crankcase, distance pieces, etc) while the cylinders are dismantled. Assembled cylinders are delivered to the site separately and installed. The vendor is advised to offer all site supervision work for cylinder installation as a closed price. 


\subsection{Auxiliaries and accessories}

\subsubsection{Lubrication system}

API 614 is typically applied only to reciprocating compressor trains involving a large turbine driver and gear unit [11]. The optimum oil system includes two oil pumps (for special purpose machines as per API 676), both sized at least $20 \%$ over that required for current operation (two motor driven identical pumps with run down tanks, or a well known crank shaft driven main oil pump; supplying UPS power for one pump is not acceptable alternative), a dual removable bundle shell and tube oil coolers (TEMA C) and double oil filters with a removable element and stainless steel piping.

\subsubsection{Coupling}

Because of special design of the reciprocating machine, the potential exists for torsional resonance and torsional fatigue failure [2]. Coupling is the best available option for modification to tune the system. The available coupling configurations are: 1- high torsional stiffness coupling (this is the optimum if allowed by torsional analysis); 2- flexible coupling (more elasticity and damping and more maintenance); 3- a direct forged flanged rigid connection (no coupling), with a single bearing motor. Coupling for big machines shall be as per API 671.

\subsubsection{Coolant system}

Liquids should never form inside the cylinder [10-12]. Liquid contributes to poor reliability, can cause high impact velocities, can lead to the stressing of valve moving elements (slugging) and reduce the lubrication effectiveness. For any application, a good-sized suction drum with a drain provision shall be in order [12]. It may be a part of the pulsation control, if properly done. The cylinder cooling system must be monitored and controlled. The coolant inlet temperature should be between $6{ }^{\circ} \mathrm{C}$ and $16{ }^{\circ} \mathrm{C}$ above the inlet gas temperature [12]. For exotic gases or operations near critical areas, much care also needs to be taken to make sure the thermodynamic model is proper.

\subsubsection{Pulsation control}

The optimum technique is to dissipate less energy rather than rely on special solutions, such as orifices to control pulsation levels [16]. Acoustic reviews shall be performed for the design and guarantee points, as well as all other operating cases and the combinations of pressures, speeds and load steps [13]. Pulsation can also alter the timing of the valve motion and decrease efficiency and reliability [5]. The preliminary optimum value for the maximum allowable peak to peak is recommended as Eq. (1), in SI units, where P1 and PL are a percentage of the average absolute pressure (\%) and the line pressure (Bara), respectively.

$$
\mathrm{P}_{1}(\%)=\frac{6}{\left(P_{L}\right)^{1 / 3}}
$$

\subsubsection{Pulsation shaking forces}

The reduction of pressure pulsation can be accompanied by an increase in shaking forces (or unbalanced forces) [17]. It illustrates that shaking forces shall 
be determined and controlled and piping and vessels properly supported. The margin of separation between the mechanical natural frequency (MNF) of the system (including piping and bottles) and excitation frequency is $20 \%$ and the MNF shall be greater than 2.4 times the maximum run speed $[11,18]$. If these limits are not met, the force response (including stress analysis) is required. The cylinder gas forces (also called frame stretch or cylinder stretch force) can be a significant source of excitation (they can cause high frequency vibration on the bottles and piping close to the compressor) and lead to excessive pulsation bottle vibration, even if the pulsation shaking forces meet limits. Flow induced pulsation is rarely seen [17]. API 618 Design Approach 3 and less rigorous analysis to control pulsation and shaking (unbalanced) force levels and avoiding mechanical resonance can result in an optimized design [17]. The pulsation and vibration analysis report includes Time Domain (TD) and Frequency Domain (FD) simulations, Time Domain (TD) plots of key forces and pressure pulsation, dynamic pressure drop, models including mounting details (mounting plate, bolts, localized skid, etc) and shell flexibility (nozzle connection flexibility), calculated cylinder stretch forces, the mode shape of bottles and piping and compressor stiffness assumption (compressor frame modelled as a flexible support) [18].

\subsubsection{Coolers}

The inter stage cooler and the after cooler should be sized carefully. An undersized cooler can cause excessive pressure drop and power loss. Pulsation and shaking force studies are necessary in order to avoid a vibration problem in the cooler. An increased cooler cross section area to decrease pressure drop can cause a significant increase of shaking force and cooler vibration. Secondary volumes may be studied to reduce this vibration, however, in some cases this solution cannot reduce vibration, and modifications to the recycle line are required in order to significantly lower shaking [17].

\subsubsection{Torsional analysis}

Typically, for a reciprocating compressor, the lateral natural frequencies will be positioned well above significant torsional natural frequencies, so lateral critical studies are not required. A stress analysis should be performed if the torsional excitation falls close to the torsional natural frequency, in order to ensure that the resonance will not be harmful for the system [18]. The torsional vibration analysis report should include data used in the mass elastic system, a display of force versus speed, torsional critical speeds, deflection (mode shape diagram), worst case design, upset condition results (such as valve failure, start up, short circuit, electrical network faults, etc) and how the input data variance will affect the results (sensitivity analysis) [18]. Continuous operation at torsional resonance should be avoided. Changing the load sequence could help to reduce torsional vibration. Full load shutdowns should be avoided. Torsional vibration should be measured and verified during the performance test. Synchronous motors or systems started on a frequency basis need more care (they definitely need a transient torsional start up analysis). The electric machine (driver) shaft 
diameter should be equal to or greater than the reciprocating compressor crankshaft diameter.

\subsubsection{Flywheel and irregularity}

For all reciprocating compressors, the flywheel is mandatory to regulate variable reciprocating torques. The irregularity degree for mechanical component reliable operation is a maximum of $2 \%$. This value is the minimum requirement for all compressors. Generally, in accordance with specific requirements for drivers (especially current pulsation for electric motors), torsional vibration results, etc, a lower irregularity value is required. It is recommended to obtain $1 \%$ for special purpose units. The optimum irregularity value is between $1-1.5 \%$.

\subsubsection{Dynamic package analysis}

The dynamic package analysis shall include modelling and simulation of the foundation at the same time. The accuracy of this analysis is strongly influenced by the design of the foundation, especially for pile installation [18]. It is even more important for packages mounted on offshore platforms, Floating Production Storage Offloading Vessels (FPSO), modules mounted on steel structures, new and unproven skid design and where the local soil conditions are suspect. A skid lifting study (including lifting lug details and a calculations review) is necessary. A transit study and environmental loading analysis are also recommended [18].

\subsubsection{Barring device}

When a compressor is stopped for an extended time, it should be turned around a quarter turn every week by a barring device. A manual barring device can be used for a small compressor. A pneumatic barring device should be used for a compressor rated over $750 \mathrm{KW}$, which is without an area classification problem or intermittent power availability and is preferred technically [11].

\subsubsection{Special tools}

The optimum check list for special tools [11] for big machines is as follows: 1bearing extractor; 2- piston extractor; 3- valve extractor; 4- piston fit up tool; 5hydraulic tightening system; 6- crosshead assembling tool; 7- special lifting tools; 8- partition plate assembling tools; 9- mandrels for wear bands. For special tools, toolboxes are required and they should be delivered with the main machines, in separate and tagged boxes.

\subsubsection{Package general arrangement}

Layout is often complex and compromise must be made between the support requirements, process requirements, vibration/pulsation conditions, access and maintenance. The optimum configuration is to install local panels near to the compressor package (around $200 \mathrm{~mm}$ away from package), but on a separate skid (frame), which is installed on a foundation in order to avoid vibration damage. For maintenance, consider spool removal and avoid support removal. Access is required in front of the cylinder for cylinder piston dismantling and the non-drive end of the compressor. Attention should be paid to the minimum elevation requirement of the pulsation bottle suction flange to keep suction line "No Pocket". 


\subsubsection{Piping analysis}

The thermal piping design often requires that flexibility be added to the system, which is counter to requirement for more support and increased stiffness to meet vibration design. These analyses should be conducted by the same party in order to optimize design iteration and result in an overall optimized system. Piping thermal analysis is necessary [18], especially when the coolers are off-skid, there are multiple compressors on a common header, there is an extremely cold ambient temperature, or the operation is over a very wide range of conditions.

\subsubsection{Cylinder lubrication system}

Using the proper type of lubricant, as well as establishing the proper lubricant rates for the cylinder and packing, can be most important for machine reliability [10]. The life of the compressor valves, piston rings, rider bands, and pressure packing can all be significantly affected by the type and quality of lubrication used. Too much or the wrong type of lubrication can increase the effects of valve stiction (viscous adhesion) and result in a reduction in reliability. A reliable lubrication system with an optimum type and rate of lubrication should be selected [10].

\subsection{Performance}

\subsubsection{Expected pressure drops}

The preliminary optimum pressure drop values are pulsation dampeners: $1 \%$ pressure and intercooler: 0,70 bar. The use of orifice plates, especially on highspeed single-acts, can contribute to significant pressure drops [13].

\subsubsection{Rod load and pin reversal details}

Maximum rod load is recommended to be less than $80 \%$ of the allowable rod load. The duration and peak magnitude load of the rod reversal should not be less than $15^{\circ}$ of the crank angle and $3 \%$ of the actual combined load [11] in the opposite direction, respectively, and should be checked for all possible operating cases $[13,19]$ (especially low suction and part load).

\subsubsection{Highest expected discharge temperature}

High discharge temperatures cause problems with lubrication cooking and valve deterioration [12] and should be reviewed at least for average and maximum suction temperatures [13]. The maximum predicted discharge temperature $[1,2,4,11]$ should not exceed $150^{\circ} \mathrm{C}$, and should not exceed $135^{\circ} \mathrm{C}$ for hydrogen rich services (MW of 12 or less). Gas discharge temperatures of less than $118^{\circ} \mathrm{C}$ tend to extend life of wearing parts [10]. This is an optimum figure.

\subsubsection{Performance curves}

The required performance curves [13] are: 1- suction pressure versus load; 2suction pressure versus flow; 3- discharge pressure versus load; 4- discharge pressure versus flow; 5 -suction pressure versus discharge pressure, per load step. Performance curves are used to safely control the unit across its defined operating range [13]. 


\subsubsection{Flow details}

Flow details are flow curves from a unit's minimum achievable flow rate to its maximum achievable flow rate, in specified increments [13]. An alternative is flow versus discharge pressure plots of specific suction pressures (more compact and common when suction pressure variation is limited).

\subsubsection{Load step curvature}

A review of the steepness of the load curves can quickly identify which load steps (and where) are quite steep in nature, and thus small changes in pressure can have significant changes in load and flow. Often, steep load curves may indicate improper sizing of cylinders and units with steep load step curves can also prove difficult to automate and tune [13].

\subsubsection{Machine shop run test}

The shop mechanical run test [11] is the first test after the manufacture of a machine and the last test before delivery. The shop test results, including vibration, may seem to have limited usefulness because the supporting structure and operating condition of the machine are different with final site installation; however this test is a unique opportunity to find defects in the design and manufacturing phases while the machine is still in the fabrication shop. Generally, larger power per compressor throw leads to higher dynamic forces and $370 \mathrm{KW}$ or more per throw is a high-risk machine [20]. It is justified and optimum to record and analyze shop run test measurements (a Kalman filter can be used to optimally evaluate machine dynamic characteristics based on measured data).

\section{Conclusion}

The optimum configuration of reciprocating compressors, with regard to component design and selection, commercial points, auxiliary and accessories, performance and reliability, are addressed in this paper.

\section{References}

[1] Heinz P. Bloch, Compressor and Modern Process Application, John Wiley and Sons, 2006.

[2] Heinz P. Bloch, A Practical Guide To Compressor Technology, Second Edition, John Wiley and Sons, 2006.

[3] W. A. Griffith, E. B. Flanagan, Online Continuous Monitoring of Mechanical Condition and Performance For Critical Reciprocating Compressors, Proceeding of the 30th Turbo-machinery Symposium, Texas A\&M University, Houston, TX, 2001.

[4] Heinz P. Bloch and John J. Hoefner, Reciprocating Compressors Operation \& Maintenance, Gulf Publishing Company, 1996.

[5] S. Foreman, Compressor Valves and Unloaders for Reciprocating Compressors - An OEM's Perspective, Dresser-Rand Technology Paper, http://www.dresser-rand.com/e-tech/recip.asp. 
[6] Steve Chaykosky, Resolution of a Compressor Valve Failure: A Case Study, Dresser-Rand Technology Report, Dresser-Rand Technology Paper, http://www.dresser-rand.com/e-tech/recip.asp.

[7] Massimo Schiavone, Evaluation of The Flow Coefficient of Cylinder Valves, Compressor Tech Two, pp. 48-50, April 2007.

[8] Robin S. Wilson, Reciprocating Compressor: Reliability Improvement Focusing on Compressor Valves, Piston and Sealing Technology, Compressor Optimization Conference, Aberdeen, 30-31 January 2007.

[9] Steven M. Schultheis, Charles A. Lickteig, Robert Parchewsky, Reciprocating Compressor Condition Monitoring, Proceeding of the Thirty Sixth Turbomachinery, pp 107-113, 2007.

[10] Stephen M. Leonard, Increasing the Increase Reliability of Reciprocating Hydrogen Compressors, Hydrocarbon Processing, pp. 67-74, January 1996.

[11] Reciprocating Compressor for Petroleum, Chemical and Gas Service Industries, API 618 5th edition, December 2007.

[12] Royce N. Brown, Compressors Selection and Sizing, Third Edition, Gulf Publishing, 2005.

[13] Dwayne Hickman, Specifying Required Performance When Purchasing Reciprocating Compressor - Part I, II, Compressor Tech Two, August September-October 2007.

[14] Enzo Giacomelli, Fabio Falciani, Guido Volterrani, Riccardo Fani, Leonardo Galli, Simulation of Cylinder Valves For Reciprocating Compressors, Proceeding of ESDA 2006, 8th Biennial ASME Conference on Engineering Systems Design and Analysis, July 4-7, 2006, Torino, Italy.

[15] Ian Cameron, Thomassen Prescience Pays Dividends, Compressor Tech Two, pp 12-13, November 2007.

[16] A. Eijk, J.P.M. Smeulers, L.E. Blodgett, A.J. Smalley, Improvements And Extensive to API 618 Related To Pulsation And Mechanical Response Studies, The Resip - A State of Art Compressor, European Forum for Reciprocating Compressor, Dresden, 4-5 Nov. 1999.

[17] Brain C. Howes, Shelley D. Greenfield, Guideline in Pulsation Studies for Reciprocating Compressors, Proceeding of IPC 02, 4th International Pipeline Conference, Calgary, Alberta, Canada, Sep. 29-Oct.3, 2002.

[18] Shelley Greenfeld and Kelly Eberle, New API Standard 618 (5 TH ED.) and its Impact on Reciprocating Compressor Package Design - Part I, II and III, Compressor Tech Two, June-July-August 2008.

[19] Alberto Guilherme Fagundes, Nelmo Furtado Fernandes, Jose Eduardo Caux, On-line Monitoring of Reciprocating Compressors, NPRA Maintenance Conference, San Antonio, May 25-28, 2004.

[20] Kelly Eberle and Chris Harper, Dynamic Analysis Of Reciprocating Compressors On FPSO Topside Modules - Part I \& II, Compressor Tech Two, pp 10-16 and pp 42-48, April \& May 2007. 